\title{
Streptococcus parasanguinis
}

National Cancer Institute

\section{Source}

National Cancer Institute. Streptococcus parasanguinis. NCI Thesaurus. Code C86800.

A species of facultatively anaerobic, Gram positive, cocci shaped bacteria in the phylum Firmicutes. This species is alpha hemolytic, alpha glucosidase and arginine deaminase positive and negative for sialidase, urease, and catalase. It can ferment lactose, and raffinose but not mannitol, sorbitol, or inulin. S. parasanguinis is found in normal human oral and respiratory flora but is a pathogen that can cause endocarditis if it colonizes the blood. 